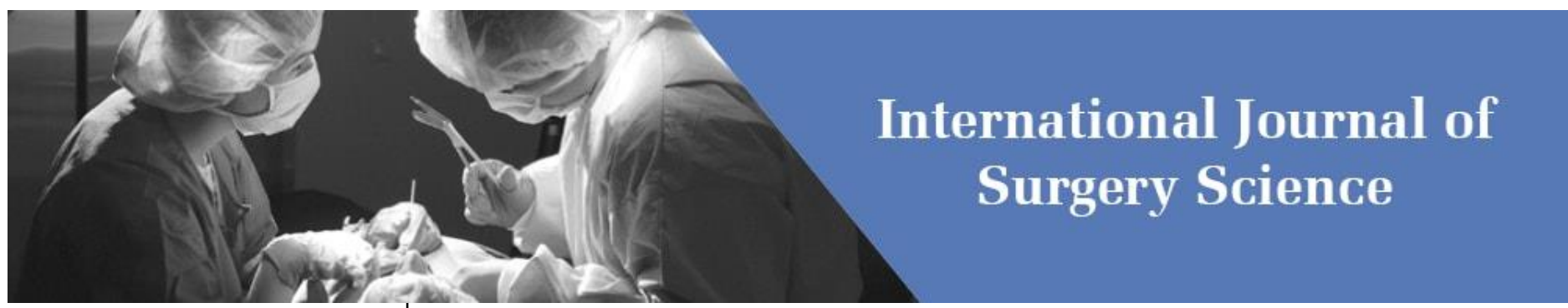

E-ISSN: 2616-3470

P-ISSN: 2616-3462

(C) Surgery Science

www.surgeryscience.com

2019; 3(4): 510-513

Received: 10-08-2019

Accepted: 12-09-2019

Avinash Tinaikar

Assistant Professor, Department of General Surgery, Karwar Institute of Medical Sciences, Karwar,

Karnataka, India

Vinay Naik

Senior Resident, Department of General Surgery, Karwar Institute of Medical Sciences, Karwar,

Karnataka, India
Corresponding Author:

Vinay Naik

Senior Resident, Department of

General Surgery, Karwar Institute

of Medical Sciences, Karwar,

Karnataka, India

\section{An observational comparative study between RIPASA \& modified Alvarado scoring in the diagnosis of acute appendicitis}

\author{
Avinash Tinaikar and Vinay Naik
}

DOI: https://doi.org/10.33545/surgery.2019.v3.i4i.293

\section{Abstract}

Background: Acute appendicitis is one of the most common surgical emergencies. Different techniques and number of scoring systems have been used for aiding in early diagnosis of acute appendicitis and its prompt management of which Alvarado score is the most popular. So we retrospectively applied and compared Alvarado and RIPASA score in the diagnosis of acute appendicitis in Indian population.

Materials and Methods: We compared retrospectively RIPASA and Alvarado scoring system by applying them to 85 patients in between June 2018 to November 2019. Both scores were calculated for patients who presented with right iliac fossa pain during the study period. Sensitivity, specificity, positive predictive value (PPV), negative predictive (NPV), diagnostic accuracy for RIPASA \& Alvarado system was done.

Results: The sensitivity and specificity of RIPASA score were $91.78 \%$ and $66.66 \%$ respectively. The sensitivity and specificity of Alvarado score were $64.38 \%$ and $58.33 \%$ respectively.

The diagnostic accuracy of RIPASA scoring system is $88.23 \%$ and that of Alvarado scoring system is $63.52 \%$. The results indicate that the RIPASA scoring system is a better diagnostic tool for the diagnosis of acute appendicitis $(p<0.001)$.

Conclusion: RIPASA scoring system is accurate, more convenient, and specific scoring system for Indian population than Alvarado scoring system.

Keywords: Acute appendicitis, RIPASA score, Alvarado score, sensitivity, accuracy

\section{Introduction}

Acute appendicitis is one of the most common cause of acute abdomen that mostly requires surgery and is usually diagnosed using clinical history, physical examination findings, and a few laboratory tests ${ }^{[1,2]}$. A differential diagnosis of acute appendicitis must include virtually every acute process within the abdomen. Some of the differential diagnosis are equally associated with other urgent clinical syndromes like ectopic pregnancy. Appendicitis has a very high and significant morbidity, which is increased with diagnostic delay. Advanced radiological imaging methods such as ultrasonography and computed tomography (CT) are often resorted to for making a quick and accurate diagnosis ${ }^{[3,4]}$. Despite all these facilities, the rate of negative or unnecessary appendectomy due to reduced diagnostic accuracy has gone up to approximately $30 \%{ }^{[2]}$. Except USG and CT for appendicitis, several clinical systems have been developed to aid in the diagnosis of acute appendicitis. A large number of scoring systems have been used for aiding in early diagnosis of acute appendicitis and it's used in prompt management. These scores make use of clinical history, physical examination, laboratory findings and many other parameters. The Raja Isteri Pengiran Anak Saleha Appendicitis (RIPASA) and Alvarado score are new diagnostic scoring systems developed for the diagnosis of Acute Appendicitis and has been shown to have significantly higher sensitivity, specificity and diagnostic accuracy. Modified Alvarado score was given by M.Kalan et al. in 1994 where patients were scored out of 9 points ${ }^{[5]}$. The RIPASA scoring system includes more parameters than Alvarado system and the latter did not contain certain parameters such as age, gender, duration of symptoms prior to presentation. The RIPASA Score is a new diagnostic scoring system developed for the diagnosis of Acute Appendicitis and has been shown to have significantly higher sensitivity, specificity and diagnostic accuracy compared to Alvarado Score, particularly when it was applied to Asian population ${ }^{[6]}$. 
There are very few studies ${ }^{[7,8,9]}$ conducted on comparison of RIPASA score and modified Alvarado score in global context. Hence the present study intended to study on Evaluation of the usefulness of the RIPASA scoring system and comparison of the RIPASA and the modified Alvarado scoring system in the diagnosis of acute appendicitis among patients admitted in general surgery wards at Karwar Institute of Medical Sciences, Karwar, Karnataka

\section{Material and Methods}

The present study was a retrospective; institution based observational study, conducted among 85 patients including males and females undergoing emergency appendicectomy in Department of General Surgery, Karwar Institute of Medical Sciences, Karwar from June 2018 - November 2019. Patients with Right iliac fossa pain, with suspicion of acute appendicitis and undergoing surgery were considered for this study. Patients with appendicular lump, evidence of generalized peritonitis, evidence of acute confusing state, dementia, septic shock, gynecological \& urological diseases on clinical ground were excluded from this study. A total of 85 patients qualified for the study during the study period. All the 85 patients were scored as per Alvarado and RIPASA scoring system; Alvarado score contained 7 parameters, whereas RIPASA Score contained 18 parameters. Then depending on the clinical details and investigation, RIPASA scoring system and Modified Alvarado Score System has been administered with corroboration of Histopathological report. The RIPASA scoring system and Modified Alvarado Score System are described below:

Table 1: RIPASA Scoring System

\begin{tabular}{|l|l|}
\hline Patients & Score \\
\hline Demography & \\
Sex:- & \\
Male & 1.0 \\
Female & 0.5 \\
Age:- & \\
Age <40 years & 1.0 \\
Age >40 years & 0.5 \\
\hline Symptoms:- & \\
Right Iliac Fossa (RIF ) pain & 0.5 \\
Migration of pain to RIF & 0.5 \\
Anorexia & 1.0 \\
Nausea and vomiting & 1.0 \\
Duration of symptoms <48 hours & 1.0 \\
Duration of symptoms >48 hours & 0.5 \\
\hline Signs:- & \\
RIF tenderness & 1.0 \\
Guarding & 2.0 \\
Rebound tenderness & 1.0 \\
Rovsing's sign & 2.0 \\
Fever & 1.0 \\
\hline Laboratory Investigations:- & \\
Raised WBC count & 1.0 \\
Negative urinalysis & 1.0 \\
\hline Additional Score & \\
Foreign NRIC & \\
\hline Total & \\
\hline Score <5 - Unlikely to be appendicitis & \\
5-7.5 - Low Probability to be appendicitis & \\
7.5-12 - Digh Probability to be appendicitis & \\
>12 & \\
& \\
\hline
\end{tabular}

Table 2: Modified Alvarado Score System

\begin{tabular}{|l|c|}
\hline \multicolumn{1}{|c|}{ Parameter } & Score \\
\hline 1. Migratory RIF pain & 1 \\
2. Anorexia & 1 \\
3. Nausea / vomiting & 1 \\
4. Tenderness in RIF & 2 \\
5. Rebound tenderness & 1 \\
6. Fever $>37.5^{\circ} \mathrm{C}$ & 1 \\
7. Leucocytosis & 2 \\
\hline \multicolumn{1}{|c|}{ Total Score } & 9 \\
\hline
\end{tabular}

Score $<5$ - Unlikely to be appendicitis

5-6 - Low Probability to be appendicitis

6-7 - High Probability to be appendicitis

$>8$ - Definite appendicitis

All the quantitative parameters were described through descriptive statistics such as mean and standard deviation. The scores were assigned to various risk factors based on the probability of the occurrence of event and total scores were computed for all the risk factors. Sensitivity (true positive rate), specificity (test result will be negative when the disease is not present), Positive Predictive Value (probability that the disease is present), Negative Predictive Value (disease is not present when the test is negative) of the scoring system will be estimated by comparing the threshold level of score with surgical findings and histopathology findings. Receiver operating characteristic (ROC) curve were used for delineating threshold score levels. ROC curve compares the diagnostic performance of two or more tests.

\section{Results}

Table 3: Distribution of patients with respect to age group.

\begin{tabular}{|c|c|c|}
\hline Age in years & No. of Patients & Percentage \\
\hline$<20$ & 21 & 24.71 \\
\hline $21-30$ & 32 & 37.65 \\
\hline $31-40$ & 19 & 22.35 \\
\hline $41-50$ & 9 & 10.59 \\
\hline $51-60$ & 4 & 4.70 \\
\hline Total & 85 & 100 \\
\hline
\end{tabular}

The age distribution in the study sample involved upto 60 years of age, majority of the patients belonged to 21-30 years age group.

Table 4: Distribution of patient with respect to gender

\begin{tabular}{|c|c|c|}
\hline Gender & No. of patients & Percentage \\
\hline Male & 58 & 68.23 \\
\hline Female & 27 & 31.77 \\
\hline Total & 85 & 100 \\
\hline
\end{tabular}

Out of 85 patients included in the study, majority of the patients in the study group were males $(68.23 \%)$.

Table 5: Distribution of patients with respect to RIPASA score

\begin{tabular}{|c|c|c|}
\hline RIPASA & No. of patients & Percentage \\
\hline$\geq 7.5$ & 71 & 83.52 \\
\hline$<7.5$ & 14 & 16.48 \\
\hline Total & 85 & 100 \\
\hline
\end{tabular}

The subject were scored according to RIPASA system and were categorized into high probability group if the score was equal to or more than 7.5 and low probability group if the score was less than 7.5.Most of the patients scored equal to or more than 7.5 $(83.52 \%)$. 
Table 6: Distribution of patients with respect to Alvarado score

\begin{tabular}{|c|c|c|}
\hline Alvarado & No. of patients & Percentage \\
\hline$\geq 5$ & 52 & 61.18 \\
\hline$<5$ & 33 & 38.82 \\
\hline Total & 85 & 100 \\
\hline
\end{tabular}

The subjects were also scored according to Alvarado system, according to Alvarado system only $61.18 \%$ of the study population were categorized as high probability of acute appendicitis as against $83.52 \%$ according to RIPASA system

Table 7: Distribution of patients based on HPE report

\begin{tabular}{|c|c|c|}
\hline HPE report & No. of patients & Percentage \\
\hline Positive & 73 & 85.88 \\
\hline Negative & 23 & 14.12 \\
\hline Total & 85 & 100 \\
\hline
\end{tabular}

Diagnosis of 85 patients were confirmed by HPE .73 patients were confirmed as acute appendicitis , 12 patients turned out to be negative for acute appendicitis in HPE resulting in negative appendectomy rate of $14.12 \%$

Table 8: Comparison of RIPASA score and HPE report

\begin{tabular}{|c|c|c|c|}
\hline \multirow{2}{*}{ RIPASA } & \multicolumn{2}{|c|}{ HPE Report } & \multirow{2}{*}{ Total } \\
\cline { 2 - 3 } & Positive & Negative & \\
\hline$\geq 7.5$ & 67 & 4 & 71 \\
\hline$<7.5$ & 6 & 8 & 14 \\
\hline Total & 73 & 12 & 85 \\
\hline
\end{tabular}

$\mathrm{P}<0.001$ (0.000004) chi square test: significant

Among 73 patients whose HPE report was positive for appendicitis, 67 patients had RIPASA score $\geq 7.5$, with $p$ score of $<0.001$ as per chi square test, which is statistically significant.

Table 9: Comparison of Alvarado score and HPE report

\begin{tabular}{|c|c|c|c|}
\hline \multirow{2}{*}{ Modified Alvarado Score } & \multicolumn{2}{|c|}{ HPE Report } & \multirow{2}{*}{ Total } \\
\cline { 2 - 3 } & Positive & Negative & 52 \\
\hline$\geq 5$ & 47 & 5 & 5 \\
\hline$<5$ & 26 & 7 & 33 \\
\hline Total & 73 & 12 & 85 \\
\hline
\end{tabular}

p> 0.001 (0.239264), chi square test: not significant

Among 73 patients whose HPE report was positive for appendicitis, 47 patients had modified Alvarado score $\geq 5$, with p score of $>0.001$ as per chi square test, which statistically not significant.

Table 10: Correlation of RIPASA score, MASS and Histopathology reports

\begin{tabular}{|c|c|c|c|}
\hline \multicolumn{2}{|r|}{ Scoring } & RIPASA & MASS \\
\hline \multirow{5}{*}{ Observation } & True positive & 67 & 47 \\
\hline & False positive & 6 & 26 \\
\hline & False negative & 4 & 5 \\
\hline & True Negative & 8 & 7 \\
\hline & Total & 85 & 85 \\
\hline \multirow{6}{*}{ Correlation (\%) } & Sensitivity & 91.78 & 64.38 \\
\hline & Specificity & 66.66 & 58.33 \\
\hline & Positive predictive value & 94.36 & 90.38 \\
\hline & Negative predictive value & 57.14 & 21.21 \\
\hline & Accuracy & 88.23 & 63.52 \\
\hline & $\mathrm{P}$ value & $<0.001$ & $>0.24$ \\
\hline
\end{tabular}

The table 10 showed comparison of RIPASA Scoring system and modified Alvarado score which revealed that RIPASA scoring system in the present study had sensitivity of $91.78 \%$, specificity of $66.66 \%$, positive predictive value $94.36 \%$, negative predictive value $57.14 \%$, diagnostic accuracy $88.23 \%$ whereas modified Alvarado score had sensitivity of $64.38 \%$, specificity of $58.33 \%$, positive predictive value of $90.38 \%$, negative predictive value of $21.21 \%$, diagnostic accuracy of $63.52 \%$.

\section{Discussion}

Acute appendicitis is one of the most common surgical emergencies, the evaluation of which is mainly based on history and clinical findings and they are the most important parameters in reaching a diagnosis of acute appendicitis. Despite of all this making a quick and accurate diagnosis of acute appendicitis can be difficult. Late or incorrect diagnosis of acute appendicitis leads to aggravation of the existing inflammation, resulting in serious complications including appendicular perforation, peritonitis, intra-abdominal abscess, and sepsis, with an increase in morbidity and mortality of patients. The diagnosis in children with acute appendicitis may be difficult due to atypical clinical features and also in elderly and females of reproductive age due to a wide range of differential diagnoses ${ }^{[10]}$. In such cases, use of advanced radiological examinations such as $\mathrm{CT}$ may have now become necessary. CT is also an expensive procedure raising the cost of healthcare and patients are exposed to radiation. There are studies in the literature reporting that unnecessary CT scans lead to unnecessary appendectomies in patients with early low-grade appendicitis which can be resolved spontaneously with antibiotics therapy and this means that such patients are burdened with surgical risks [7]. However, sometimes the diagnosis of acute appendicitis can only be made based on the intra-operative macroscopic appearance of the appendix tissue and the histopathological examination of the removed appendectomy material ${ }^{[4]}$. Since the problems of correctly diagnosing acute appendicitis have been experienced for a very long time in almost all surgical outpatient departments worldwide, many scoring systems have been developed for the diagnosis of acute appendicitis including Alvarado, modified Alvarado, appendicitis inflammatory response score, Ohmann score, and Lintula score. However, because all these scoring systems produced different results in different ethnic groups and had rather low sensitivities and specificities, there came a need arose to devise new systems. Developed in 2010 and started to be used more widely thereafter, the RIPASA score is an inexpensive, very easy to use and highly reliable quantitative scoring system that enables to make a correct and early diagnosis of acute appendicitis and to significantly reduce the negative appendectomy rate. RIPASA score is simple and easy to use as a quantitative scoring system and most of the included parameters can easily be obtained from good clinical history and examination it also included urine analysis which can be easily performed.

Our study compared sensitivity and specificity between Alvarado Scoring System with that of RIPASA. Sensitivity or true positive rate is the proportion of actual positives which is correctly identified that is the percentage of sick people who are correctly identified as having the condition. Here it is found that the RIPASA score was considerably better than Alvarado score in correctly diagnosing acute appendicitis. Using the RIPASA score, $91.78 \%$ of patients who actually had acute appendicitis were correctly diagnosed and placed in the high probability group (RIPASA score > 7.5), compared to only $64.38 \%$ when using the Alvarado score on the same population sample. Again, the diagnostic accuracy of RIPASA was $88.23 \%$ and Alvarado Score was $63.52 \%$ indicating that the RIPASA score is a much better diagnostic tool for the diagnosis of acute appendicitis. 
Table 11: Comparative Analysis of Sensitivity and Specificity with Other Global Studies

\begin{tabular}{|c|c|c|c|c|}
\hline \multirow{2}{*}{ Study } & \multicolumn{2}{|c|}{ RIPASA } & \multicolumn{2}{c|}{ Alvarado } \\
\cline { 2 - 5 } & Sensitivity (\%) & Specificity (\%) & Sensitivity (\%) & Specificity (\%) \\
\hline Chong et al. $2010^{[2]}$ & 98 & 81.32 & $68.32 \%$ & $87.9 \%$ \\
\hline Alnjadat et al. ${ }^{[8]}$ & 93.2 & 61.8 & 73.7 & 68.6 \\
\hline Erdem et al. $2013^{[11]}$ & 100 & 28 & 82 & 75 \\
\hline Reyes-Garcia et al. $2012^{[9]}$ & 91.2 & 84.6 & 89.5 & 69.2 \\
\hline Present study & 91.78 & 66.66 & 64.38 & 58.33 \\
\hline
\end{tabular}

Table 12: Comparative analysis of the Diagnostic Accuracy

\begin{tabular}{|c|c|c|}
\hline \multirow{2}{*}{ Study } & \multicolumn{2}{|c|}{ Diagnostic Accuracy (\%) } \\
\hline & RIPASA & Alvarado \\
\hline Chong et al. 2010 [2] & 91.83 & 86.5 \\
\hline Alnjadat et.al ${ }^{[8]}$ & 91.5 & 74.3 \\
\hline Erdem et al. $2013^{[11]}$ & 77 & 80 \\
\hline Present Study & 88.23 & 63.52 \\
\hline
\end{tabular}

In our study, most of the patients with a RIPASA score $\geq 7.5$ were diagnosed with catarrhal stage appendicitis. The rest of the patients in this group had appendicitis of a more advanced pathological stage. The patients with a RIPASA score 12 and over had suppurative-or gangrenous-stage appendicitis.

Appendicitis was found in the histopathological examinations of most of the patients who had RIPASA scores higher than 7.5. When this is taken into consideration together with the above mentioned disadvantages of CT, we think a CT scan is not necessary for patients with a RIPASA score 7.5 and over.

This type of a practice will fully justify the validity of the existence of a RIPASA scoring system. In this context, it will be useful to inform physicians, particularly those working in rural hospitals without a CT unit, about the necessity to use the RIPASA scoring system more frequently.

\section{Conclusion}

The RIPASA score is simple scoring system with high sensitivity and specificity for the diagnosis of acute appendicitis .RIPASA score is currently a better diagnostic scoring system for diagnosis of acute appendicitis compared to Alvarado score particularly in Indian population. Making a correct and prompt diagnosis of acute appendicitis including its possible pathological stage is possible with the RIPASA score, which is easily obtained using simple clinical and laboratory data, without a need of unwanted admissions and expensive imaging studies like CT scan.

\section{References}

1. Karami MY, Niakan H, Zadebagheri N, Mardani P, Shayan Z, Deilami I. Which one is better? Comparison of the acute inflammatory response, raja isteri pengiran anak saleha appendicitis and alvarado scoring systems, Annals of Coloproctology. 2017; 3(6):227-231.

2. Chong CF, Adi MIW, Thien A. Development of the RIPASA score: A new appendicitis scoring system for the diagnosis of acute appendicitis. Singapore Medical Journal. 2010; 51(3):220-225.

3. do Nascimento RR, Souza JCG, Alexandre VB, Kock KS, and Kestering DM, Association between the Alvarado score and surgical and histopathological findings in acute appendicitis. Revista do Col'egio Brasileiro de Cirurgioes. 2018; 45(5):1901.

4. Sousa-Rodrigues CF, Rocha AC, Rodrigues AK, Barbosa FT, Ramos FW, Valoes SH. Correlation between the Alvarado Scale and the macroscopic aspect of the appendix in patients with appendicitis. Revista do Col'egio Brasileiro de Cirurgĩoes. 2014; 41(5):336-339.

5. Gupta M, Virdi VS, Agnihotri L, Mandial V. Evaluation of Modified Alvarado Score and Ultrasonography for the Diagnosis of Acute Appendicitis. IJSR. 2016; 5(3):2166-68.

6. Antel J, Rivera L, Landenberg B, Halm G, Fatava MA. Brown CVR Clinical diagnostic pathway for acute Appendicitis: prospective Validation JAM Coll Surg. 2006; 203(6):849-56.

7. Chong CF, Thien A, Mackie AJ, Tin AS, Tripathi S, Ahmad MA, et al. Comparison of RIPASA and Alvarado scores for the diagnosis of acute appendicitis. Singapore Med J. 2011; 52(5):340-5

8. Alnjadat I, Abdallah B. Alvarado versus RIPASA score in diagnosing acute appendicitis. Rawal Med J. 2013. 38(2):147-51.

9. Reyes-Garcia, Zaldiver-Ramirez FR, Creuz-Martinez R, Sandoval Martinez MD, Gutierrez- Banda CA, Athie Gutierrez C. diagnostic accuracy of the RIPASA score for the diagnosis of acute appendicitis: comparative analysis with the Modified Alvarado score. Cir Gen. 2012; 34(2):101-6.

10. Gunes Tatar I, Yilmaz KB, Sahin A, Aydin H, Akinci M, Hekimoglu B. Evaluation of clinical alvarado scoring system and CT criteria in the diagnosis of acute appendicitis, Radiology Research and Practice, vol. 2016, Article ID 9739385, 2016, 6.

11. Erdem H, Çetinkunar S, Daş K, Alvarado, Eskelinen, Ohhmann, Raja Isteri Pengiran Anak Saleha Appendicitis scores for diagnosis of acute appendicitis. World J Gastroenterol. 2013; 19(47):9057-9062. 